\title{
Use of Quinolones in Bull Semen Extenders to Reduce Sperm Deoxyribonucleic Acid Damage
}

\author{
${ }^{1}$ Clara Gonzalez-Marin, ${ }^{1}$ Michael Eduard Kjelland, \\ ${ }^{2}$ Rosa Roy, ${ }^{2}$ Carmen Lopez-Fernandez, ${ }^{3}$ Jose Luis Fernandez, \\ ${ }^{1}$ Juan Fernando Moreno and ${ }^{2}$ Jaime Gosalvez \\ ${ }^{1}$ Department of Research and Development, Sexing Technologies, \\ 22575 State Highway 6 South, Navasota, TX 77868, USA \\ ${ }^{2}$ Department of Genetics Unit Biology, University Autonoma of Madrid, Darwin \# 2, 28049 Madrid, Spain \\ ${ }^{3}$ Department of Genetics, A Coruna Universty Hospital, As Xubias 84, 15006, A Coruna, Spain
}

Received 2012-07-29, Revised 2012-12-17; Accepted 2012-12-29

\begin{abstract}
Cryopreserved sperm samples from Holstein bulls $(n=20)$ were examined for bacterial presence and Sperm DNA Fragmentation (SDF) dynamics. SDF was assessed after thawing (T0) and at 4, 24 and $48 \mathrm{~h}$ of incubation $\left(37^{\circ} \mathrm{C}\right)$ and the rate of SDF (r-SDF), as an estimator of the DNA degradation over time, was calculated. Two groups of bulls were identified based on the presence or absence of bacteria: One group $(n=10)$ had a readily detectable bacterial presence, while the other group $(n=10)$ had an undetectable bacterial presence. Differences in the SDF at $\mathrm{T} 0$ were not observed between these two groups. However, statistically different results were found after $24 \mathrm{~h}$ of incubation at $37^{\circ} \mathrm{C}$ (Kaplan-Meier estimator; Log-Rank Matel-Cox, $\mathrm{p}<0.000$ ). To test the direct effect of bacteria on SDF, sperm samples from 6 bulls without bacteria were inoculated with $5 \mu \mathrm{L}$ of sperm that consistently exhibited a bacterial presence at $48 \mathrm{~h}$ of incubation at $37^{\circ} \mathrm{C}$. Differences were observed in the r-SDF between the control and the inoculated samples. In a parallel experiment, sperm samples from 10 bulls were treated with $1 \mu \mathrm{g} \mathrm{mL}{ }^{-1}$ of ciprofloxacin at T0. Differences in the r-SDF $(\mathrm{p}>0.05)$ were not detected between the control and the quinolone treated sample for those samples without bacteria. However, differences $(p<0.000)$ in SDF were observed for quinolone treated samples that previously presented bacteria. Incubation of sealed straws showed that bacterial contamination occurred prior to cryopreservation. These results call attention to three points: (1) sperm samples were in contact with bacteria before cryopreservation; (2) the r-SDF can be directly correlated with bacterial presence but this effect remains cryptic after thawing and (3) the r-SDF can be reduced by treating the semen samples with an adequate antibiotic such as quinolones, a finding not previously reported in the scientific literature, but important in terms of reproduction.
\end{abstract}

Keywords: Bacteria, DNA Fragmentation, Antibiotics

\section{INTRODUCTION}

The bacterial presence in semen samples could be especially problematic in situations where the ejaculates are used for artificial insemination $24 \mathrm{~h}$ or more after collection (Suarez and Pacey, 2006; Hawk, 1983). Therefore, it is of great importance that the extended shipped ejaculates maintain the highest quality upon arrival (Keck et al., 1998). The incidence of bacterial presence in the germ line can impact semen quality (Eley et al., 2005; Satta et al., 2006), also affecting the DNA molecule. Thus, Gallegos et al. (2008), demonstrated that human patients infected with Chlamydia trachomatis and Mycoplasma had 22575 State Highway 6 South, Navasota, TX 77868, USA 
increased values of sperm DNA damage compared to control individuals. In Holstein bulls, we have recently found that regular commercial straws can show traces of bacteria but after incubation these bacteria grow and have a negative impact on the sperm DNA quality by producing a decrease in the sperm DNA longevity (Gonzalez-Marin et al., 2011). Quinolones have been demonstrated as being highly efficient at interfering with bacterial growth. The aim of the present experiment was to test the possibility of modifying the rate of sperm DNA fragmentation in vitro using quinolones for those bulls' semen samples in which a bacterial presence was observed. Additionally, to demonstrate the direct relationship between the presence of bacteria and sperm DNA damage, we hypothesized that the rate of SDF observed in standard straws with an undetectable amount of bacteria, could also be modified by inoculating these samples with a small amount of semen from a sample having a detectable amount of bacteria, i.e., outright contaminating them.

\section{MATERIALS AND METHODS}

Six straws from 20 Holstein bulls were randomly selected for each bull with the criteria "straws from different ejaculates". A total of 120 straws were assessed for dynamic loss of DNA quality and bacterial presence. The dynamic loss of DNA quality over time or the rate of Sperm DNA Fragmentation (r-SDF) was estimated as the increase of SDF per time unit (hours). Cryopreserved samples were thawed by immersion in a $37^{\circ} \mathrm{C}$ water bath for 30 seconds and divided into different single doses using a Tris extender with a $\mathrm{pH}$ of 6.8 and an osmolarity balanced at $\sim 300 \mathrm{mOsm}$. All samples were prepared using fresh sperm extender containing three antibiotics (Gentamycin sulfate, Streptomycin sulfate and Tylosin $\left(\right.$ Tylan $\left.^{\circledR}\right)$. Each aliquot was diluted to a concentration of $\sim 25 \times 10^{6}$ sperm/straw $(0.5 \mathrm{cc})$.

For serial SDF assessment each aliquot was removed from incubation after $0,4,24$ and $48 \mathrm{~h}$ (T0-T48) and assessed for SDF using the Sperm-Halomax ${ }^{\circledR}$ kit (Halotech DNA, Spain) as explained elsewhere (Gosalvez et al., 2011). The threshold level for considering that a sample had a bacterial presence was established at $\geq 5$ bacteria per microscope field, when analyzed using fluorescence microscopy and a 400x magnification. Sperm and bacteria were simultaneously visualized under fluorescence microscopy after being stained with the DNA intercalating fluorochrome GelRed (Biotium, Hayward, CA, USA) 10x in anti-fading Vectashield (Vector Laboratories, Burlingame, CA, USA). A Leica DMLA model motorized epifluorescence microscope controlled with software for automatic scanning and image digitization (Leica Microsystems, Barcelona, Spain) was used. A Leica EL6000 fluorescence light source equipped with a metal halide lamp and PlanFluotar 40x objective for routine analysis and Plan-Fluotar 60x objective for image capturing were utilized.

The inoculation of bacteria into those semen samples lacking bacteria was performed to test the capacity of the bacterial presence to change the r-SDF. Immediately after thawing, six sperm samples from bulls previously classified in the group of bulls without bacteria were inoculated with $5 \mu \mathrm{L}$ of sperm that showed bacteria after $48 \mathrm{~h}$ of incubation. For each bull, a control sample lacking bacterial inoculation was analyzed. Determination of SDF for all samples was conducted after $0,4,24$ and $48 \mathrm{~h}$ of incubation.

To determine the effect of bacterial presence on SDF, 10 sperm samples from different bulls were treated with $1 \mu \mathrm{g} \mathrm{mL} \mathrm{m}^{-1}$ of ciprofloxacin directly added to the aliquot at T0. Five of the ciprofloxacin treated samples belonged to the group of bulls that presented bacteria in semen samples between $0-48 \mathrm{~h}$ of incubation at $37^{\circ} \mathrm{C}$ after thawing. The other five samples were bulls with an undetectable presence of bacteria in sperm during a similar period of incubation time. For each bull, a control sample without quinolone treatment underwent the same incubation time and temperature conditions. Samples were maintained in a $37^{\circ} \mathrm{C}$ water bath for $48 \mathrm{~h}$ and determination of SDF was conducted after $0,4,24$ and $48 \mathrm{~h}$ of incubation.

To distinguish whether the bacterial presence in the post-thaw semen sample was a result of inoculation of bacteria during the dilution process, or whether it occurred during the processing steps previous to cryopreservation, 5 sealed straws of a batch identified as currently presenting bacteria and 5 straws identified as free of bacteria were incubated at $37^{\circ} \mathrm{C}$ in a sterile laminar flow cabinet for $48 \mathrm{~h}$. The straws were placed onto a sterile $37^{\circ} \mathrm{C}$ temperate plate and covered with an aluminum box to avoid direct UV radiation. After the $48 \mathrm{~h}$ period of incubation, each straw was tested for the presence of bacteria.

The loss of sperm DNA quality over time (i.e., temporal dynamics) was compared in the different experiments using the nonparametric maximum 
likelihood Kaplan-Meier estimator. The log rank test statistic (Mantel-Cox) which compares estimates of the hazard functions between the two groups at each time interval was used (SPSS v.17.0 for Windows, SPSS Inc., Chicago, Illinois, USA). The sperm survival (an inverse estimate of the r-SDF) is represented as a varying frequency over time for those sperm which were not affected by fragmentation at each time period of analysis (Kjelland et al., 2011).

\section{RESULTS}

The baseline level (T0) of SDF was similar in all of the Holstein bull sperm samples, independently of whether they presented bacteria or not (compare T0h in Fig. 1a with 1b). The values of SDF were less than $5 \%(2.79 \% \pm 1.41 \%)$ at T0 hours. To analyze the

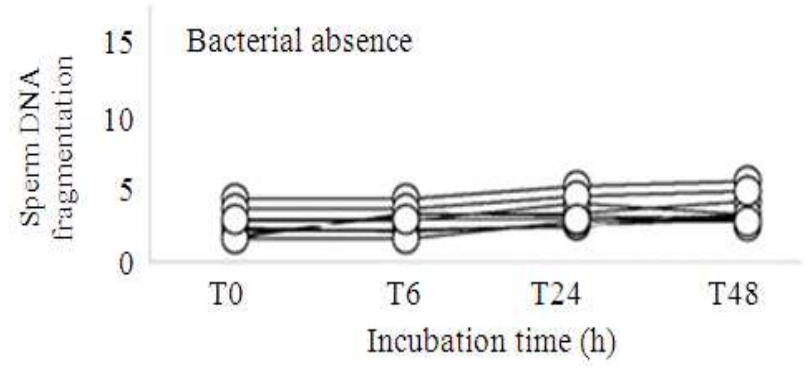

(a)

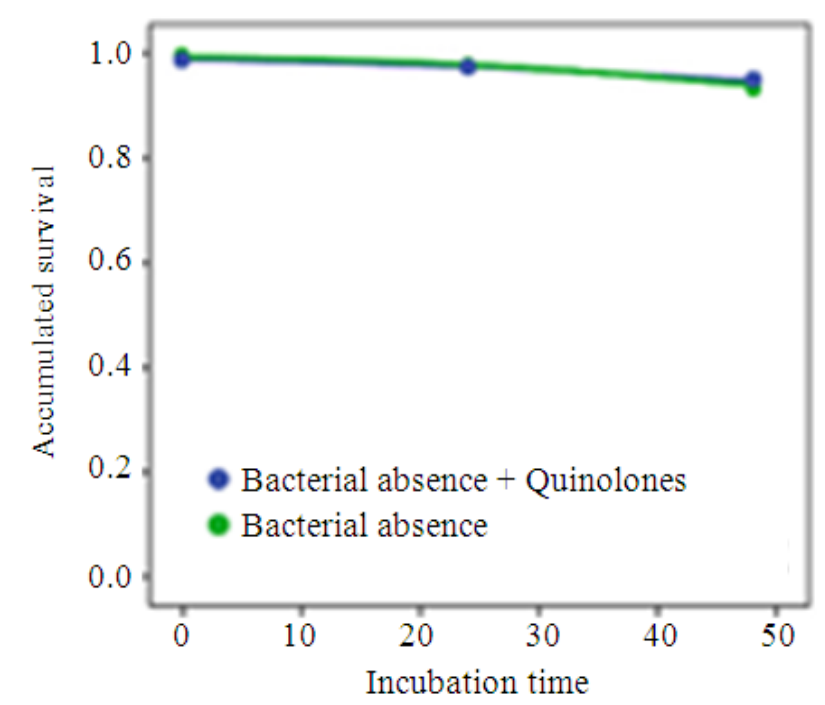

(c) influence of bacterial presence on the r-SDF, the SDF was assessed at different incubation times and the data plotted and compared according to the cluster criteria "ejaculates with bacteria versus ejaculates free of bacteria" (Fig. 1a, b). Differences in the level of DNA damage were not observed at T0 hours $(p>0.05)$. However, this situation changed as the samples were incubated at $37^{\circ} \mathrm{C}$ (compare Fig. 1a and 1b). A significantly increase in the level of sperm DNA fragmentation after $24 \mathrm{~h}$ of incubation was observed. The $\mathrm{r}-\mathrm{SDF}$ at $\mathrm{T} 24 \mathrm{~h}$ ranged from an increase in the DNA damage of about $0.6 \%$ per $h$ (arrow in Fig. 1b) to a $4 \%$ per $\mathrm{h}$ (arrow head in Fig. 1b). Kaplan-Meier estimator showed significant differences $(p<0.000)$ for the rate of SDF when straws with and without bacteria were compared.

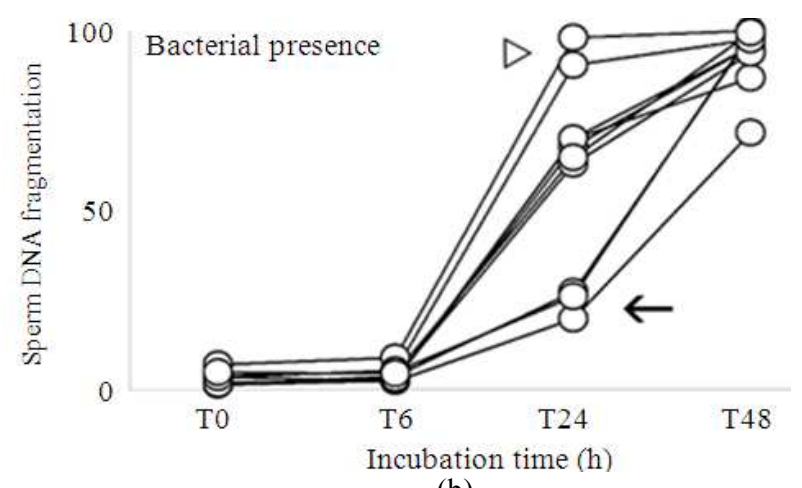

(b)

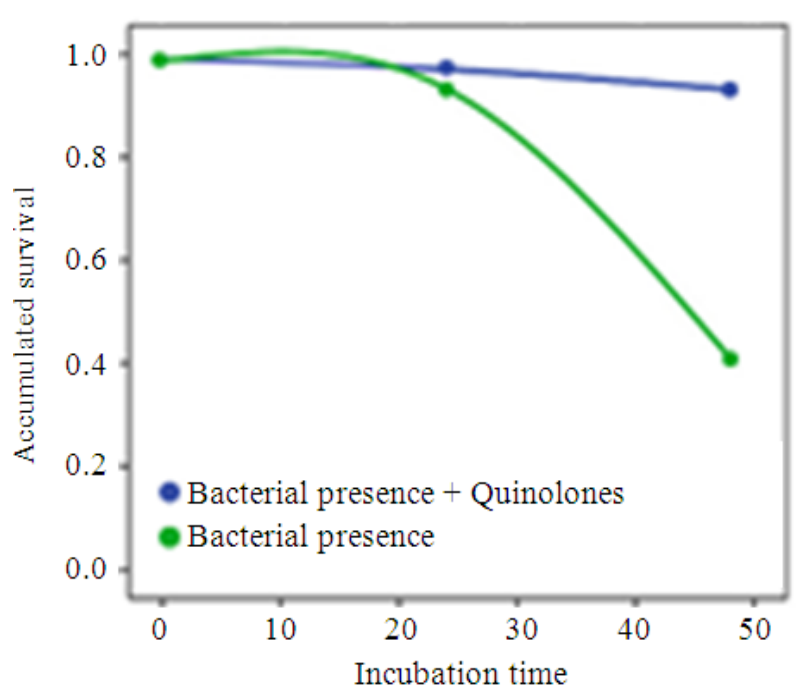

(d) 


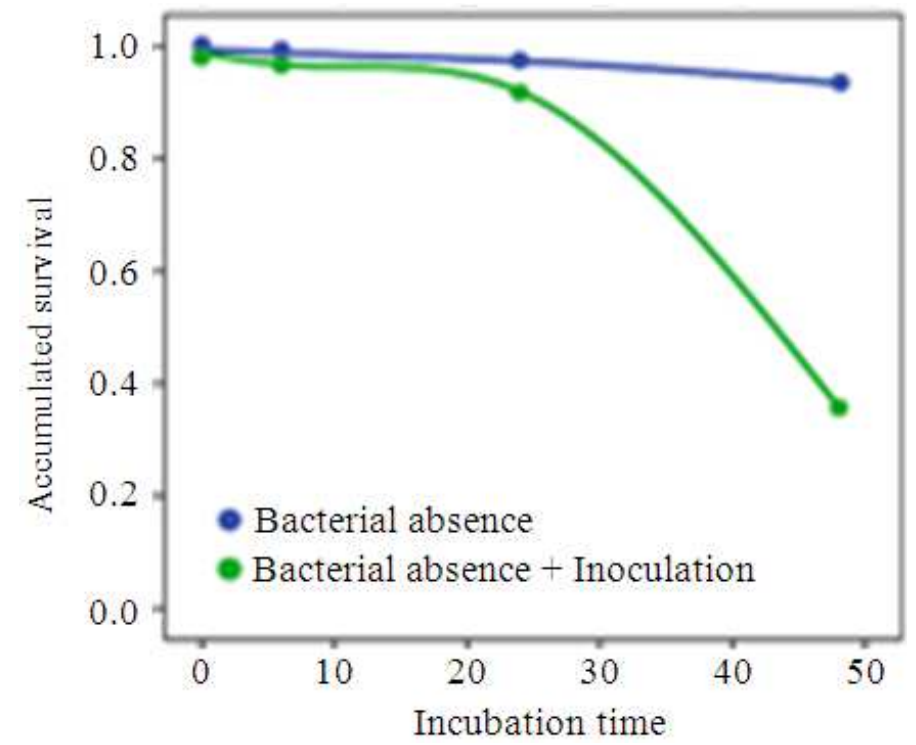

(e)

Fig. 1. Graphic representation of the dynamics of Sperm DNA Fragmentation (SDF) in samples with absence of bacteria (a) and samples where bacteria were readily present (b). Figure 1c-e shows Kaplan- Meier survival functions for the average of the sperm samples used under different experimental conditions (see text for details). (c) Bacterial absence plus quinolones (blue line) and bacterial absence (green line). (d) Bacterial presence plus quinolones (blue line) and bacterial presence (green line). (e) Bacterial absence (blue line) and bacterial absence plus bacterial inoculation (green line)

Differences in the rate of SDF were not observed $(\mathrm{p}>0.05)$ when straws without bacteria were compared to straws that did not present bacteria but had quinolones added (Fig. 1c). To test the direct effect of bacterial presence on the rate of SDF and quinolone presence, sperm samples from bulls classified in the group of individuals with bacteria were compared with aliquots of the same bulls treated with quinolones. The KaplanMeier estimator showed significant differences between both groups (Fig. 1d; $\mathbf{p}<0.000$ ).

To test the direct effect of bacteria on the r-SDF, sperm samples from 6 different bulls classified in the group of individuals without bacteria were inoculated after thawing with $5 \mathrm{uL}$ of sperm that exhibited a bacterial presence after $48 \mathrm{~h}$ of incubation. The KaplanMeier estimator showed significant differences between the groups (Fig. 1e p<0.05). Additionally, in the experiment where sealed straws were thawed and immediately incubated at $37^{\circ} \mathrm{C}$ in a sterile environment, only the cases which were pre-determined as belonging to a straw batch having a bacterial presence, showed bacteria growth. The rest of the sealed straws remained without a bacterial presence, thereby confirming that the bacteria inoculation occurred pre-cryopreservation.

\section{DISCUSSION}

The results obtained in the present investigation call attention to six major points of interest and all of them should be considered when attempting to reduce the impact of bacteria on the sperm DNA quality in order to improve the quality of bull semen samples commercially distributed in frozen straws: (1) the presence of bacteria in the semen sample occurred previously to the cryopreservation process; (2) bacteria are resistant to the antibiotic cocktail regularly used in the semen extenders or, at least, its activity is quite limited; (3) the increase in SDF is directly correlated with the presence of bacteria; (4) horizontal transmission of bacteria from samples with bacteria to samples without bacteria triggers SDF; (5) SDF can be significantly reduced or abolished by incubating sperm samples with quinolones; and (6) all of these effects are clearly detected after performing a dynamic assessment of SDF (T0-T48 h), but remains cryptic if the impact on the DNA is analyzed just after sperm thawing (T0), i.e., this is a time dependent effect.

Two important aspects need to be revised in the scenario of bull semen commercialization: (1) the bacterial presence, such as we have reported and suggested in a previous analysis (Gonzalez-Marin et al., 
2011), is a fact linked to the steps of sperm handling prior to or during the process of cryopreservation (Smole et al., 2009), but it also depends upon the bull and (2) the negative effect produced on the sperm DNA molecule by bacterial growth could easily be controlled using antibiotics such as quinolones. As far as we know, this antibiotic in not currently used to prepare semen extenders for bull straw commercialization. The current trend in semen sales indicates that the demand for ejaculates of high quality and not just limited to Bovine, is likely to continue and the role that antibiotics like quinolones can play on reducing bacterial presence could be significant in economic terms. Quinolones belong to a group of antimicrobial family which are the most frequently prescribed agents worldwide, ciprofloxacin a second generation derivate, is one of the most prescribed for humans although the fourth generation is now being tested. For specific veterinary use, there are a number of molecules (danofloxacin, difloxacin, enrofloxacin or sarafloxacin) that could be as effective as the ciprofloxacin for these purposes. However, as happens with all antibiotics, a negligent use of these molecules may provoke undesirable resistance (Nelson et al., 2007). Compared to antibiotics which target for peptidoglycans or different components of the bacterial cell wall, the activity of quinolones is mediated by the production of DNA damage via the trapping of DNA gyrase and/or topoisomerase IV on bacterial DNA (Drlica et al., 2008). Interestingly, King et al. (1997) made the first studies using different molecules included in the family of the quinolones at physiologic concentrations on human semen samples. They found that when sperm were incubated for $48 \mathrm{~h}$ in the presence of ciprofloxacin hydrochloride or doxycycline hyclate, sperm hyperactivation was decreased but did not affect overall sperm motility when tested in the physiologic range. However, using pharmacologic concentrations of ciprofloxacin hydrochloride, cefuroxime axetil and nitrofurantoin monohydrate, the authors reported an adverse effect on motility with decreased rapid progression. Cefuroxime axetil and nitrofurantoin monohydrate practically abolished sperm motility. But remarkably, the authors also reported that when used under a physiological dose, ciprofloxacin hydrochloride or ofloxacin are able to enhance sperm fertilizing capacity, as determined from the sperm penetration assay. Additionally, the authors noted that alterations in the acrosome were not found after a prolonged interaction of these compounds in the semen extender. In the particular case of dromedary camels, the addition of 400 microg $\mathrm{mL}^{-1}$, in combination with a Tris lactose extender, is capable of eliminating bacterial contamination without a negative effect on post-thaw sperm motility or acrosomal integrity (El-Bahrawy et al., 2010).

Data from our study suggest that the use of quinolones in semen extenders can arrest bacterial proliferation by direct attachment to the bacteria DNA molecule, which may be an effective alternative to traditional cell wall directed antibiotics. However, fertility trials with these quinolone molecules have not yet been conducted for the specific use in bull ejaculates and further investigations to examine the effects, if any, on other sperm characteristics should be undertaken.

\section{CONCLUSION}

Cryopreserved bull perm samples containing bacteria were in contact with bacteria before cryopreservation. When these sperm samples were cryopreserved using conventional antibiotics (gentamycin sulfate, streptomycin sulfate or Tylosin), a notably reduction in sperm DNA longevity was observed; this effect remains cryptic after thawing and sperm incubation for $24 \mathrm{~h}$ at $37^{\circ} \mathrm{C}$. Sperm DNA longevity can be increased by treating the semen samples containing bacteria with alternative antibiotics such as quinolones. These aspects are of interest in terms of promoting alternative cryopreservation strategies to increase reproductive outcome.

\section{ACKNOWLEDGEMENT}

The researchers thank Ms. F. Arroyo and A. Gosalbez for their technical help. The authors also thank Richard W. Lenz for his suggestions regarding the manuscript. This work was supported by the Ministry of Education and Science, Spain (Grant BFU2010-16738/BFI).

\section{REFERENCES}

Drlica, K., M. Malik, R.J. Kerns and X. Zhao, 2008. Quinolone-mediated bacterial death. Antimicrob. Agents Chemother, 52: 385-392. DOI: 10.1128/AAC.01617-06

El-Bahrawy, K.A., E. El-Sayed, El-Hassanein and Y.M. Kamel, 2010. Comparison of Gentamicin and Ciprofloxacin in dromedary camel's semn extender. World J. Agric. Sci., 6: 419-424.

Eley, A., S. Hosseinzadeh, H. Hakimi, I. Geary and A.A. Pacey, 2005. Apoptosis of ejaculated human sperm is induced by co-incubation with Chlamydia trachomatis lipopolysaccharide. Hum. Reprod, 20: 2601-2607. DOI: 10.1093/humrep/dei082 
Gallegos, G., B. Ramos, R. Santiso, V. Goyanes and J. Gosalvez et al., 2008. Sperm DNA fragmentation in infertile men with genitourinary infection by Chlamydia trachomatis and Mycoplasma. Fertil. Steril., 90: 328-334. DOI: 10.1016/j.fertnstert.2007.06.035

Gonzalez-Marin, C., R. Roy, C. Lopez-Fernandez, B. Diez and M.J. Carabano et al., 2011. Bacteria in bovine semen can increase sperm DNA fragmentation rates: A kinetic experimental approach. Anim. Reprod. Sci., 123: 139-148. DOI: 10.1016/j.anireprosci.2010.11.014

Gosalvez, J., M.A. Ramirez, C. Lopez-Fernandez, F. Crespo and K.M. Evans et al., 2011. Sex-sorted bovine spermatozoa and DNA damage: I. Static features. Theriogenology, 75: 206-211. DOI: 10.1016/j.theriogenology.2010.08.006

Hawk, H.W., 1983. Sperm survival and transport in the female reproductive tract. J. Dairy. Sci., 66: 26452660. DOI: $10.3168 /$ jds.S0022-0302(83)82138-9

Keck, C., C. Gerber-Schafer, A. Clad, C. Wilhelm and M. Breckwoldt, 1998. Seminal tract infections: Impact on male fertility and treatment options. Hum. Reprod. Update, 4: 891-903. DOI: 10.1093/humupd/4.6.891

King, K., P.J. Chan, W.C. Patton and A. King, 1997. Antibiotics: Effect on cryopreserved-thawed human sperm motility in vitro. Fertil. Steril., 67: 11461151. DOI: $10.1016 / \mathrm{S} 0015-0282(97) 81453-7$
Kjelland, M.E., C. Gonzalez-Marin, J. Gosalvez, C. Lopez-Fernandez and R.W. Lenz et al., 2011. DNA fragmentation kinetics and postthaw motility of flow cytometric-sorted white-tailed deer sperm. J. Anim. Sci., 89: 3996-4006. DOI: 10.2527/jas.2011-4014

Nelson, J.M., T.M. Chiller, J.H. Powers and F.J. Angulo, 2007. Fluoroquinolone-resistant Campylobacter species and the withdrawal of fluoroquinolones from use in poultry: A public health success story. Clin. Infect. Dis., 44: 977-980. DOI: $10.1086 / 512369$

Satta, A., A. Stivala, A. Garozzo, A. Morello and A. Perdichizzi et al., 2006. Experimental Chlamydia trachomatis infection causes apoptosis in human sperm. Hum. Reprod., 21: 134-137. DOI: 10.1093/humrep/dei269

Smole, I., A. Thomann, J. Frey and V. Perreten, 2009. Repression of common bull sperm flora and in vitro impairment of sperm motility with Pseudomonas aeruginosa introduced by contaminated lubricant. Reprod. Domest. Anim., 45: 737-742. PMID: 19144031

Suarez, S.S. and A.A. Pacey, 2006. Sperm transport in the female reproductive tract. Hum. Reprod. Update, 12: 23-37. DOI: 10.1093/humupd/dmi047 